\title{
Management of chronic pain after hernia repair
}

This article was published in the following Dove Press journal: Journal of Pain Research

\section{Kristoffer Andresen Jacob Rosenberg}

Department of Surgery, Herlev Hospital, University of Copenhagen, Copenhagen, Denmark
Correspondence: Kristoffer Andresen Department of Surgery, Herlev Hospital, University of Copenhagen, Herlev Ringvej 75, 2730 Herlev, Denmark

Tel +45 25468424

Fax +45 38684009

Email Kristofferandresen@gmail.com

\begin{abstract}
Chronic pain following inguinal hernia repair is a common problem and feared complication. Up to $16 \%$ of people experience chronic pain following the repair of a groin hernia. The aim of this review was to provide an overview of treatment strategies for patients with chronic pain following inguinal hernia repair based on best practice guidelines and current clinical routines. The optimal management of chronic pain following inguinal hernia surgery should begin with a thorough clinical examination to rule out other causes of chronic pain and to rule out a recurrence. A scaled approach to treatment is recommended. Initially, watchful waiting can be tried if it can be tolerated by the patient and then systemic painkillers, escalating to blocks, and surgery as the final option. Surgery should include mesh removal and triple neurectomy following anterior approaches or mesh and tack removal following a posterior approach. The diagnosis and treatment strategies should be performed by or discussed with experts in the field. Keywords: inguinal hernia, chronic pain, management, surgery, pharmacology, radio frequency
\end{abstract}

\section{Introduction}

Postoperative chronic pain is a special entity within the domain of chronic pain. Chronic postoperative pain occurs following numerous kinds of surgery, from amputations to thoracotomies to inguinal hernia surgery. The chronic pain after inguinal hernia repair has been extensively studied; however, the management is still difficult. Around the globe, millions of groin hernia repairs are conducted annually ${ }^{1}$ and $8 \%-16 \%$ of these patients experience chronic pain to a degree that impairs their daily lives 6 months postoperatively. ${ }^{2,3} \mathrm{~A}$ few percent of these patients experience disabling pain, and due to the large number of groin hernia repairs, the number of patients with disabling pain and discomfort is an important clinical problem. ${ }^{4}$ Depending on the degree of pain and the impact on daily activities, an evidence-based treatment option should be offered. A problem with some of the current evidence, based on research in chronic pain after groin hernia repair, is the use of different assessment methods and different lengths of follow-up. Surgeons, scientists, and pain specialists use different assessment methods, ie, researchers use different questionnaires in order to assess chronic pain in research projects, and this limits comparability.

The aim of this review is to provide an overview of treatment strategies for patients with chronic pain following inguinal hernia repair based on best practice guidelines and clinical routines. 


\section{Groin hernia repairs and pain}

For the initial repair of groin hernia, recommendations include use of a mesh. ${ }^{5,6}$ Dedicated centers have shown good results with nonmesh-based techniques, ${ }^{7}$ but the overall recommendation for routine care includes use of a mesh. Globally, the most used types of mesh-based repairs are the Lichtenstein open repair and one of the variations of the laparoscopic repair. ${ }^{8}$ The Lichtenstein repair is known to cause discomfort and disabling pain in some patients. ${ }^{3,9}$ The laparoscopic techniques seem to lower the risk of chronic pain but still cause chronic pain in some people. ${ }^{10}$

Several potential causes of pain have been investigated for these repair methods. The role of the meshes has been investigated, and the weight of the mesh $\left(\mathrm{g} / \mathrm{m}^{2}\right)$ has been hypothesized to influence the risk of pain. Heavyweight meshes have been compared with lightweight meshes but demonstrated no advantages for laparoscopic repairs. ${ }^{11}$ However, the lightweight meshes seem to lower the risk of chronic pain following the Lichtenstein repair technique. ${ }^{12}$ Besides meshes, the choice of fixation method for the mesh can influence the risk of pain. Glue has been demonstrated to reduce the risk of chronic pain in open mesh-based repairs, ${ }^{13,14}$ but the advantage of glue could not be found in a recent nationwide study of the laparoscopic repair. ${ }^{10}$

When operating inguinal hernias, there is a risk of causing injuries that will cause pain. If there is injury to tissue, it can cause nociceptive pain, and damage to nerves can cause neuropathic pain. In order to prevent or minimize the risks of pain, surgeons always take care when performing repairs and handle tissue gently. Peroperatively, the role of identification and/or resection of nerves has been investigated in order to minimize the occurrence of chronic pain. The nerves can be in the way when placing the mesh, can be caught or injured during fixation, and can be injured by accident or by dissection in the operative field. It is recommended, in the recently published World Guidelines for inguinal hernia repair, to identify the nerves but not to do a planned resection; however, if the nerves are in the way when placing the mesh, a "pragmatic resection" is recommended. ${ }^{5}$

\section{Definitions and types of pain}

Chronic (postoperative) pain has been defined as pain lasting at least 2-3 months (after surgery), but modifications are proposed to this timeframe. ${ }^{15,16} \mathrm{~A}$ group of experts in hernia surgery and chronic pain has suggested modifying the definition for chronic pain after hernia repair as pain lasting at least 6 months after operation. ${ }^{4}$ The reason for this extended period of time is because the inflammation around the mesh is still ongoing after 3 months, and there is a chance that some patients will improve substantially from 3 to 6 months postoperatively.

\section{Pathogenesis}

The cause of chronic pain following inguinal hernia surgery can be multifactorial and often it is not possible to point to a distinct reason for the pain in a specific patient. Pain can be caused by perioperative injury to nerves or nerves that are being stuck and damaged by sutures or perforated by fixation devices such as tacks. Nerves can also be trapped in a "meshoma", which is when the mesh has shrunk with folding and wrinkling making a ball of mesh material, the so-called meshoma. ${ }^{17}$ It is reasonable to imagine that some shrinking of the mesh, which is seen in most patients, can also entrap a nerve and thereby cause chronic pain. Pain can also be caused by the inflammation process around the mesh. It is sometimes not possible to diagnose the specific cause of pain in the individual patient. Furthermore, the distinction between neuropathic pain (pain caused by damage to nerves) and nociceptive pain (pain caused by the release of substances due to tissue damage or damage to organs) can be difficult. Some patients might have groin pain from other sources than their hernia repair such as hip pathology or pubalgia. Furthermore, some patients might have received a hernia repair due to these other pathologies, resulting in symptoms similar to a hernia.

When investigating the patient with chronic postoperative pain, it is important to look for a possible recurrence since the recurrence could be the reason for pain or discomfort. If a recurrence is present, it is indicated to repair the recurrence and hopefully that will relieve the complaints and discomfort of the patient. However, care should be taken when repairing recurrent hernias if there is also signs of neuropathic pain, since the patient might at the same time have damaged nerves. If there are damaged nerves, repair of the recurrence might not solve the problem for, and it is therefore recommended to also consider a neurectomy at the same time.

\section{Preoperative and early postoperative pain}

A patient's preoperative level of pain affects the risk of developing chronic pain following surgery. A large questionnaire study found that preoperative pain increased the risk of postoperative pain by a factor $1.5 .{ }^{10}$ However, preoperative pain is not a contraindication for surgery, but patients should be informed of the increased risk. Not only does the preoperative pain influence the risk of postoperative pain, but also there 
are strong indications that early high-intensity pain after surgery is a risk factor for developing chronic pain. ${ }^{15}$ Therefore, a standardized and effective postoperative analgesic regime could be recommended following inguinal hernia repair.

\section{Assessment of pain and severity}

When assessing the degree of pain in a research context, it is important to use validated questionnaires and questionnaires that also evaluate the impact on activities of daily living, such as driving, walking, and standing. ${ }^{18-20}$

For day-to-day clinical work and assessment of patients, it is time-consuming to use these questionnaires and a thorough history as well as clinical examination should be used. It is important to have experience with chronic pain after inguinal hernia surgery when assessing these patients. Some surgeons use a surgical marker to "map" the pain on the patient, with pluses indicating pain, zeros indicating no pain, and minuses meaning numb area. ${ }^{21}$ The groin, abdomen, thigh, and genitals should be investigated in order to locate the extent of the painful area. Photos of this "map" can be taken and then compared with the same investigation after treatment or shared with colleagues and experts in order to plan the best management strategy for the patient. ${ }^{22}$ The pain map can provide an indication of which nerves might be involved in the chronic pain pathogenesis. "Pain maps" are sometimes shared in a closed Facebook group called International Hernia Collaboration Group, which now has more than 3,000 members. Here, surgeons discuss clinical cases and the best strategy to treat patients with, eg, chronic postoperative pain.

A more formal testing can be done with the quantitative sensory testing (QST). QST uses a range of methods to investigate pain by pressure, pain, cold, pin prick, etc. QST is time-consuming and not applicable for most routine clinical settings. $^{2}$

\section{Interventions and management of chronic pain}

Authors of a recent review aimed to characterize management and treatment strategies for chronic postoperative pain; however, the authors of the review concluded that there was a lack of evidence, making it impossible to draw firm conclusions regarding effectiveness or harm. ${ }^{23}$ A problem might be to investigate all interventions to treat all postoperative chronic pain conditions, and it is likely that each of the different procedures resulting in chronic pain needs to have their own specific treatment and diagnostic algorithm.
For chronic pain following inguinal hernia surgery, a range of interventions can be used to treat or manage the chronic pain. Interventions include watchful waiting, pharmacological treatments, local anesthetic blocks, sensory stimulation or ablation of nerves, and surgery (mesh removal, reoperation for recurrence, and neurectomy). ${ }^{24}$

\section{Watchful waiting}

First-line management of chronic pain following inguinal hernia repair is to examine the patient and explain the condition. Since pain is declining with time after surgery, a watchful strategy can be tried in the beginning. ${ }^{25}$ However, a lifestyle change to sedentary activities is not recommended, ${ }^{26}$ and chronic pain can severely affect quality of life. ${ }^{27}$ Basic analgesics can be used in the period of watchful waiting. ${ }^{25}$ If there are no improvements in the condition after a few months, then systematic pharmacological interventions should be tried, preferably in collaboration with pain specialists. If the pain is bearable, it is import to inform the patient that pain will, for many patients, diminish with time. ${ }^{28}$

\section{Pharmacological interventions}

Systematic pharmacological analgesics are used in the postoperative phase to reduce the acute pain. For chronic pain at the later stages, after 3-6 months, depending on definition, pharmacological interventions can be tried as a first-line treatment, using nonsteroidal anti-inflammatory drugs, gabapentinoids, tricyclic antidepressants, selective serotonin reuptake inhibitor/serotonin-norepinephrine reuptake inhibitor, and/or conventional analgesics. ${ }^{29}$ Topical administration of analgesics has also been considered. Patches with lidocaine and capsaicin for local application have been tested in randomized studies. Unfortunately, the studies have not been able to demonstrate a benefit, and these patches are therefore not recommended for the treatment of chronic groin pain. ${ }^{30,31}$

Prevention of chronic pain with the use of preoperatively administered pharmacological interventions has been investigated, ${ }^{32}$ the hypothesis being that it could be possible to diminish postoperative pain and prevent central sensitization. ${ }^{29}$ A Cochrane review including 40 randomized clinical trials concluded, cautiously, that preoperatively administered ketamine may reduce the risk of chronic pain but that there is no evidence for recommending gabapentinoids, pregabalin, or other drugs for prevention of chronic postoperative pain. ${ }^{33} \mathrm{At}$ this point, evidence is not strong enough to systematically recommend preoperative analgesics for the prevention of chronic pain, but future studies might prove it beneficial, maybe especially in patients at high risk of developing chronic pain. 


\section{Injections}

Injections with local anesthetics have been tested and are used for both the relief of pain and the diagnostic purposes in order to identify which nerves are involved in neuropathic pain. Fixed point injections do not have an effect on chronic postoperative pain compared with placebo treatment. ${ }^{34}$ In a study, it was tested to use injections at tender points, meaning that the point of injection was tailored to each patient depending on where in the groin the pain was. ${ }^{35}$ The study demonstrated some effect indicating that the peripheral nerves were involved in the chronic pain, but unfortunately, the effect did not last. ${ }^{35} \mathrm{~A}$ recent study on injections with a mix of lidocaine, corticosteroids, and hyaluronic acid has investigated the use of up to three repeated injections over 6 weeks in order to treat chronic pain compared with surgical neurectomy. The study demonstrated a $22 \%$ success rate, which was lower than the $71 \%$ success rate for the neurectomy group. ${ }^{36}$ In the study, $4 \%$ minor complications were found for injections. The injections were safe and minimally invasive, and the authors concluded that it should be considered to use injections before explorative surgery with neurectomy. ${ }^{36}$ Injections with alcohol can be used for neurolysis.

\section{Radiofrequency treatment}

Radiofrequency can be used to block the nerve by moderate heating $\left(40^{\circ} \mathrm{C}\right)$ that does not result in thermocoagulation or can be used at a higher intensity, thereby heating the nerve resulting in ablation of the tissue. Radiofrequency can be done either at the peripheral level or at the vertebral level. A study investigated pulsed radiofrequency at the dorsal root ganglia with heating of the nerve to $40-42^{\circ} \mathrm{C}$, demonstrating pain relief for up to 20 weeks. ${ }^{37}$ Another study investigated radiofrequency at a higher intensity, where the nerves were heated to $70-90^{\circ} \mathrm{C}$, thereby causing thermocoagulation of the nerve, and demonstrated pain relief lasting up to 12 months. ${ }^{38}$

Another option is to implant a device that stimulates the nerves (spinal cord stimulation), ${ }^{39}$ and this has been demonstrated to provide some relief up to 12 months. ${ }^{40}$ However, the current clinical use of radiofrequency or spinal implants is limited. ${ }^{41}$

\section{Surgery}

If other treatments (watchful waiting, systemic analgesics, and injections) have been tried and failed, it should be considered to operate the patients for their pain (Figure 1). Different surgical approaches can be used to remove the mesh, perform a neurectomy, or a combination of both. Neurectomy can be performed via an anterior open approach or via a posterior laparoscopic technique. Surgical strategy will be determined by the initial technique for inguinal hernia repair. Following anterior approaches, a repeated anterior approach with neurectomy and mesh removal is recommended. Following posterior approaches (ie, laparoscopy), a posterior approach is recommended with exploration of the field and removal of tacks and mesh.

If it is thought that the mesh is causing the problem, and not a recurrence, a surgical removal of the mesh should be considered..$^{25}$ The pain can be due to damage to the nerves caused by the mesh, but simply removal of the mesh might not reverse the neuropathic pain. Peroperatively, the nerves might seem normal without macroscopically visible damage, but there can still be ultrastructural damages to the nerves that will cause pain and even the removal of the mesh might not reverse the damage and the patient might still have pain. It is therefore recommended to always conduct a triple neurectomy since it is difficult if not impossible to diagnose which nerves are involved in the pain, and a subsequent surgical procedure in the scarred surgical field after mesh removal may be difficult. Thus, when performing mesh removal after a previous Lichtenstein repair, it is recommended to remove as much of the mesh as possible (preferably all mesh material) together with triple neurectomy anterior to the annulus, ie, outside of the abdominal cavity without dissection behind the transversalis fascia.

Surgical treatment of pain following a posterior approach such as laparoscopy can be divided in two methods. The first method is to remove tacks and mesh with a laparoscopic approach. The second method is a neurectomy that should be reserved for skilled hands.

The laparoscopic retroperitoneal triple neurectomy has been developed to avoid the scared field of the groin after open approaches and in order to conduct neurectomy proximal to the nerves following preperitoneal or intraperitoneal approaches. ${ }^{42}$ In a highly selected group of patients (62 out of 567 with chronic pain after inguinal hernia repair), $95 \%$ of patients experienced decrease of their pain to "manageable levels" after laparoscopic retroperitoneal triple neurectomy. ${ }^{42}$

\section{Centralization}

The treatment of patients with chronic pain following inguinal hernia surgery should be considered centralized. In Germany, dedicated hernia centers have been established. ${ }^{43}$ The specialized management of the patients with this pain should be considered as an expert area. Centralized evaluation and treatment would also allow for proper data collection and high 


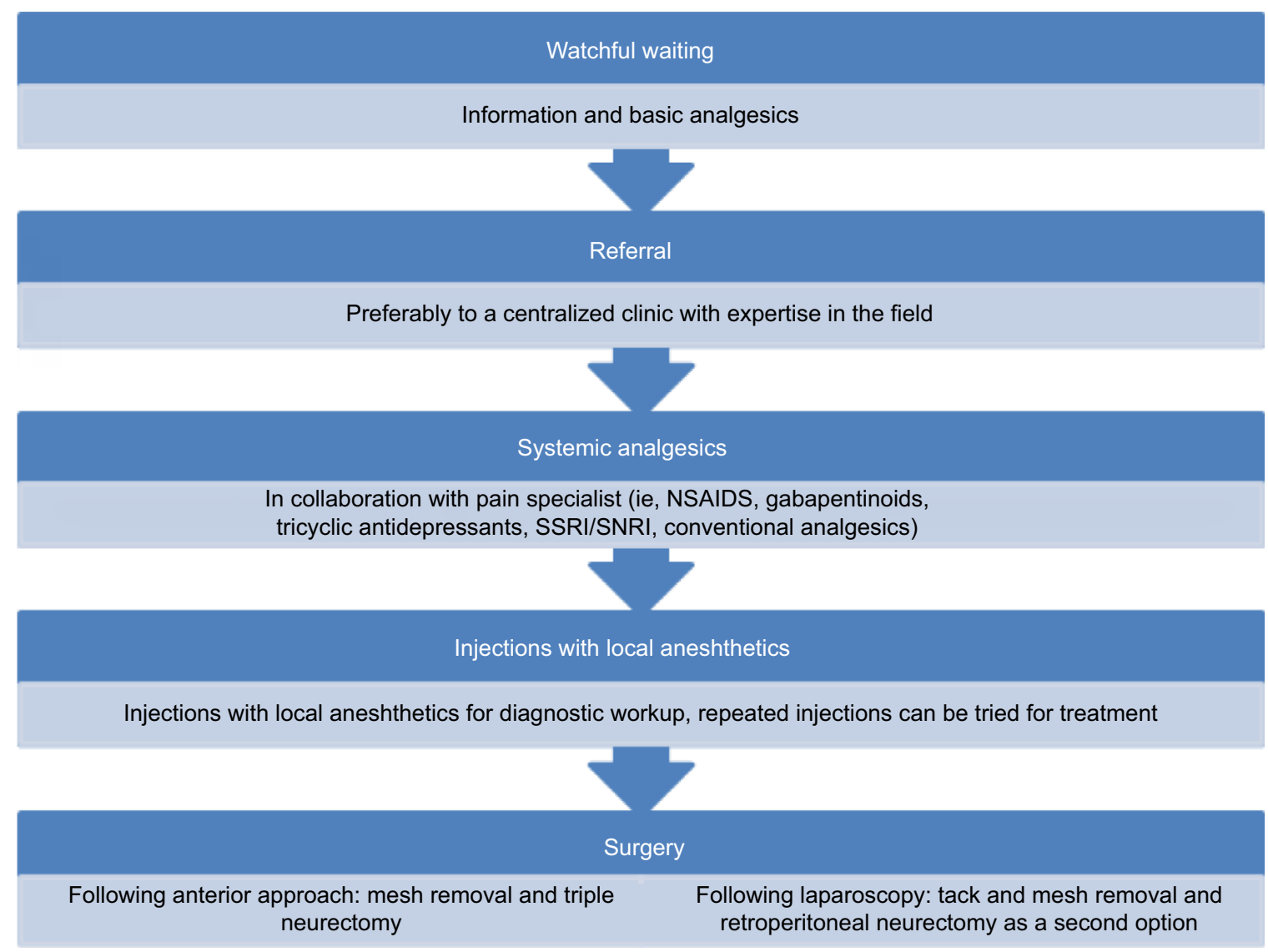

Figure I Proposed treatment options for chronic pain following inguinal hernia repair.

Note: A scaled approach is suggested if the patient's condition allows it.

Abbreviations: NSAID; nonsteroidal anti-inflammatory drug; SSRI, selective serotonin reuptake inhibitor; SNRI, serotonin-norepinephrine reuptake inhibitor.

volume research. In Denmark, these patients are all referred to one clinic, which has resulted in good data collection and a uniform treatment of the patients.

\section{Discussion}

This review aimed to provide an overview of possible strategies for the treatment of chronic pain after inguinal hernia surgery. At the moment, there is not a single treatment or management strategy that will be appropriate for all patients. Experts and expert-based guidelines recommend to use a scaled intervention, meaning that the patient should start out with watchful waiting, especially in the first 6 months, and then, systemic pain killers and if that cannot relieve the pain, injections should be tried; surgical treatment should be considered as the final option. It is also recommended to always consult a surgeon or, preferably, a regional or a national center with the expertise in chronic pain following inguinal hernia surgery since causes and treatments vary from patient to patient and because one strategy might not fit all.
Several guidelines exist in this area; however, they are mostly based on expert opinions and personal experience and less on solid high-quality evidence. ${ }^{5,25} \mathrm{~A}$ regional, national, or maybe even international setup should be in place in order to deal with the most severe and complex cases. This allows for gathering of expertise and ensures that patients are uniformly treated and receive the best standard of care for this condition. An international collaboration could be beneficial in order to share knowledge and collect data for development of treatments. It is likely that the individual surgeon does not see their own patients with chronic pain if the patients go to a different surgeon or institution. Thus, just for reoperation for recurrence, up to $25 \%$ of patients go to a different hospital than for the primary repair. ${ }^{44}$

In general, there is a high need for evidence regarding chronic postsurgical pain management, ${ }^{23}$ not only for pain following inguinal hernia surgery but also for pain following many different kinds of surgery. The treatment of chronic postoperative pain is difficult, and a simple treatment that works well for all has not been found. ${ }^{23}$ Therefore, individu- 
alized management is becoming more important, and the optimal management is what every surgeon or doctor treating these patients should aim for.

\section{Conclusion}

The optimal management of chronic pain following inguinal hernia surgery should begin with a thorough clinical examination to rule out other causes of chronic pain and to rule out a recurrence. If other causes of chronic groin pain have been excluded and the groin pain is caused by the inguinal hernia repair, a scaled approach is recommended. Initially, watchful waiting can be tried if it can be tolerated by the patient and then systemic pain killers, escalating to blocks, and surgery as the final option. Surgery should include mesh removal and triple neurectomy following anterior approaches or mesh and tack removal following a posterior approach. These procedures should be performed by experts in the field. There is a need for new developments and evidence-based guidelines, and this will require centralization of treatment in order to gather the necessary data to support future best practice guidelines.

\section{Disclosure}

The authors report no conflicts of interest in this work.

\section{References}

1. Kingsnorth A. Treating inguinal hernias. BMJ. 2004;328(7431):59-60.

2. Aasvang EK, Gmaehle E, Hansen JB, et al. Predictive risk factors for persistent postherniotomy pain. Anesthesiology. 2010;112(4):957-969.

3. Andresen K, Burcharth J, Fonnes S, et al. Chronic pain after inguinal hernia repair with the ONSTEP versus the Lichtenstein technique, results of a double-blinded multicenter randomized clinical trial. Langenbecks Arch Surg. 2017;402(2):213-218.

4. Alfieri S, Amid PK, Campanelli G, et al. International guidelines for prevention and management of post-operative chronic pain following inguinal hernia surgery. Hernia. 2011;15(3):239-249.

5. The HerniaSurge Group. International Guidelines for Groin HerniaManagement. Hernia; 2018;22(1):1-165.

6. Rosenberg J, Bisgaard T, Kehlet H, et al; Danish Hernia Database. Danish Hernia Database recommendations for the management of inguinal and femoral hernia in adults. Dan Med Bull. 2011;58(2):C4243.

7. Shouldice EB. The shouldice repair for groin hernias. Surg Clin North Am. 2003;83(5):1163-1187.

8. Fitzgibbons RJ Jr, Forse RA. Clinical practice. Groin hernias in adults. N Engl J Med. 2015;372(8):756-763.

9. Bischoff JM, Aasvang EK, Kehlet H, Werner MU. Does nerve identification during open inguinal herniorrhaphy reduce the risk of nerve damage and persistent pain? Hernia. 2012;16(5):573-577.

10. Andresen K, Fenger AQ, Burcharth J, Pommergaard HC, Rosenberg J. Mesh fixation methods and chronic pain after transabdominal preperitoneal (TAPP) inguinal hernia surgery: a comparison between fibrin sealant and tacks. Surg Endosc. 2017;31(10):4077-4084.

11. Burgmans JP, Voorbrood CE, Simmermacher RK, et al. Long-term results of a randomized double-blinded prospective trial of a lightweight (Ultrapro) versus a heavyweight mesh (Prolene) in laparoscopic total extraperitoneal inguinal hernia repair (TULP-trial). Ann Surg. 2016;263(5):862-866.
12. Sajid MS, Leaver C, Baig MK, Sains P. Systematic review and metaanalysis of the use of lightweight versus heavyweight mesh in open inguinal hernia repair. Br J Surg. 2012;99(1):29-37.

13. Colvin HS, Rao A, Cavali M, Campanelli G, Amin AI. Glue versus suture fixation of mesh during open repair of inguinal hernias: a systematic review and meta-analysis. World J Surg. 2013;37(10): 2282-2292.

14. Campanelli G, Pascual MH, Hoeferlin A, et al. Randomized, controlled, blinded trial of Tisseel/Tissucol for mesh fixation in patients undergoing Lichtenstein technique for primary inguinal hernia repair: results of the TIMELI trial. Ann Surg. 2012;255(4):650-657.

15. Werner MU, Kongsgaard UE. I. Defining persistent post-surgical pain: is an update required? Br J Anaesth. 2014;113(1):1-4.

16. Schug SA, Pogatzki-Zahn M. Chronic pain after surgery or injury. Pain Clin Updates. 2011;14(1):1-5.

17. Amid PK. Radiologic images of meshoma a new phenomenon causing chronic pain after prosthetic repair of abdominal wall hernias. JAMA Surg. 2004;139(12):1297-1298.

18. McCarthy MJ, Jonasson $\mathrm{O}$, Chang $\mathrm{CH}$, et al. Assessment of patient functional status after surgery. J Am Coll Surg. 2005;201(2):171-178.

19. Franneby U, Gunnarsson U, Andersson $M$, et al. Validation of an Inguinal Pain Questionnaire for assessment of chronic pain after groin hernia repair. Br J Surg. 2008;95(4):488-493

20. Heniford BT, Walters AL, Lincourt AE, Novitsky YW, Hope WW, Kercher KW. Comparison of generic versus specific quality-oflife scales for mesh hernia repairs. J Am Coll Surg. 2008;206(4): 638-644.

21. Chen DC, Hiatt JR, Amid PK. Operative management of refractory neuropathic inguinodynia by a laparoscopic retroperitoneal approach. JAMA Surg. 2013;148(10):962-967.

22. Álvarez R. Dermatome mapping: preoperative and postoperative assessment. In: Jacob B, Chen D, Ramshaw B, Towfigh S, editors. The SAGES Manual of Groin Pain. Cham: Springer; 2016:277-292.

23. Wylde V, Dennis J, Beswick AD, et al. Systematic review of management of chronic pain after surgery. Br J Surg. 2017;104(10):1293-1306.

24. Werner MU. Management of persistent postsurgical inguinal pain. Langenbecks Arch Surg. 2014;399(5):559-569.

25. Lange JF, Kaufmann R, Wijsmuller AR, et al. An international consensus algorithm for management of chronic postoperative inguinal pain. Hernia. 2015;19(1):33-43.

26. Hakeem A, Shanmugam V. Current trends in the diagnosis and management of post-herniorraphy chronic groin pain. World J Gastrointest Surg. 2011;3(6):73-81.

27. Courtney CA, Duffy K, Serpell MG, O’Dwyer PJ. Outcome of patients with severe chronic pain following repair of groin hernia. Br J Surg. 2002;89(10):1310-1314.

28. Oberg S, Andresen K, Rosenberg J. Gradual decline in prevalence of chronic pain after laparoscopic groin hernia repair: a nationwide crosssectional questionnaire study. In press 2018.

29. Bjurstrom MF, Nicol AL, Amid PK, Chen DC. Pain control following inguinal herniorrhaphy: current perspectives. J Pain Res. 2014;7: 277-290.

30. Bischoff JM, Ringsted TK, Petersen M, Sommer C, Uceyler N, Werner MU. A capsaicin (8\%) patch in the treatment of severe persistent inguinal postherniorrhaphy pain: a randomized, double-blind, placebo-controlled trial. PLoS One. 2014;9(10):e109144.

31. Bischoff JM, Petersen M, Uceyler N, Sommer C, Kehlet H, Werner MU. Lidocaine patch $(5 \%)$ in treatment of persistent inguinal postherniorrhaphy pain: a randomized, double-blind, placebo-controlled, crossover trial. Anesthesiology. 2013;119(6):1444-1452.

32. Clarke H, Bonin RP, Orser BA, Englesakis M, Wijeysundera DN, Katz $\mathrm{J}$. The prevention of chronic postsurgical pain using gabapentin and pregabalin: a combined systematic review and meta-analysis. Anesth Analg. 2012;115(2):428-442.

33. Chaparro LE, Smith SA, Moore RA, Wiffen PJ, Gilron I. Pharmacotherapy for the prevention of chronic pain after surgery in adults. Cochrane Database Syst Rev. 2013;7:CD008307. 
34. Bischoff JM, Koscielniak-Nielsen ZJ, Kehlet H, Werner MU. Ultrasound-guided ilioinguinal/iliohypogastric nerve blocks for persistent inguinal postherniorrhaphy pain: a randomized, double-blind, placebocontrolled, crossover trial. Anesth Analg. 2012;114(6):1323-1329.

35. Wijayasinghe N, Ringsted TK, Bischoff JM, Kehlet H, Werner MU. The role of peripheral afferents in persistent inguinal postherniorrhaphy pain: a randomized, double-blind, placebo-controlled, crossover trial of ultrasound-guided tender point blockade. Br J Anaesth. 2016;116(6):829-837.

36. Verhagen T, Loos MJA, Scheltinga MRM, Roumen RMH. The GroinPain trial: a randomized controlled trial of injection therapy versus neurectomy for postherniorraphy inguinal neuralgia. Ann Surg. Epub 2017 Apr 26.

37. Makharita MY, Amr YM. Pulsed radiofrequency for chronic inguinal neuralgia. Pain Physician. 2015;18(2):E147-E155.

38. Kastler A, Aubry S, Piccand V, Hadjidekov G, Tiberghien F, Kastler B. Radiofrequency neurolysis versus local nerve infiltration in 42 patients with refractory chronic inguinal neuralgia. Pain Physician. 2012;15(3) $237-244$
39. Schu S, Gulve A, ElDabe S, et al. Spinal cord stimulation of the dorsal root ganglion for groin pain-a retrospective review. Pain Pract. 2015;15(4):293-299.

40. Levine AB, Steven DA, Parrent AG, MacDougall KW. Successful long-term nerve root stimulation for chronic neuropathic pain: a real world, single center Canadian experience. Pain Physician. 2017;20(2): 95-106.

41. Schwartz JS, Strosberg DS, Renton DB. Chronic groin pain following anterior hernia surgery. In: Jacob BP, Chen DC, Ramshaw B, Towfigh S, editors. The SAGES Manual of Groin Pain. Cham, Springer; 2016:211-219.

42. Moore AM, Bjurstrom MF, Hiatt JR, Amid PK, Chen DC. Efficacy of retroperitoneal triple neurectomy for refractory neuropathic inguinodynia. Am J Surg. 2016;212(6):1126-1132.

43. Kockerling F, Berger D, Jost JO. What is a certified hernia center? The example of the German hernia society and German society of general and visceral surgery. Front Surg. 2014;1:26.

44. Nolsøe A, Andresen K, Rosenberg J. Repair of recurrent hernia is often performed at a different clinic. Hernia. 2016;20(6):783-787.

\section{Journal of Pain Research}

\section{Publish your work in this journal}

The Journal of Pain Research is an international, peer reviewed, open access, online journal that welcomes laboratory and clinical findings in the fields of pain research and the prevention and management of pain. Original research, reviews, symposium reports, hypothesis formation and commentaries are all considered for publication

\section{Dovepress}

The manuscript management system is completely online and includes a very quick and fair peer-review system, which is all easy to use. Visit http://www.dovepress.com/testimonials.php to read real quotes from published authors. 